\title{
Muco-cutaneous ulceration induced by low doses of methotrexate
}

Keywords: methotrexate, toxicity, cutaneous ulcerations, rheumatology, pancytopenia

\section{Introduction}

Methotrexate is a folate analog anti metabolite, which competitively and irreversibly inhibits dihydrofolate reductase. It is widely used across various specialties viz. dermatology, rheumatology, oncology, gynaecology etc. Methotrexate continues to be one of the most effective drugs in management of Psoriasis even after the advent of biologics for more than two decades. Methotrexate has many severe life threatening side effects including hepatotoxicity and pancytopenia. Methotrexate toxicity usually occurs over prolonged use or increased dosages. Cutaneous ulcerations at therapeutic doses of Methotrexate have been seldom reported. We report a case of psoriasis with psoriatic arthropathy presenting with cutaneous ulcerations of preexisting psoriatic plaques after just two doses of Tab Methotrexate $7.5 \mathrm{mg} /$ week.

\section{Case report}

A 62 years old patient of chronic plaque-type psoriasis with psoriatic arthritis symptomatic for seventeen years, presented with ulcerations of psoriatic plaques on dorsum of hands and an ulcer on lower lip of 07 days duration. Patient was being managed on cap cyclosporine for psoriatic arthritis, which had to be stopped due to rise in serum creatinine. He was therefore switched to oral Methotrexate $7.5 \mathrm{mg} /$ weekly. After taking first dose of oral Methotrexate $7.5 \mathrm{mg} /$ $\mathrm{wk}$, he started developing redness over the psoriatic plaques on the dorsum of both hands. A week later, following second dose of methotrexate, he developed erosions on the pre-existing redness over the psoriatic plaques on the dorsum of both hands along with oral mucosal ulcers. There was no history of fever, sore throat, fatigue or systemic symptoms.

Dermatological examination showed erythema and erosions of the psoriatic plaques over dorsum of both hands (Figure 1) and superficial ulcerations and erosions on mucosal surface of both lips (Figure 2). However, psoriatic plaques over other parts of body (extensor surfaces of elbows, knees, legs and feet) were in remission with no fresh psoriatic activity or ulcerations.

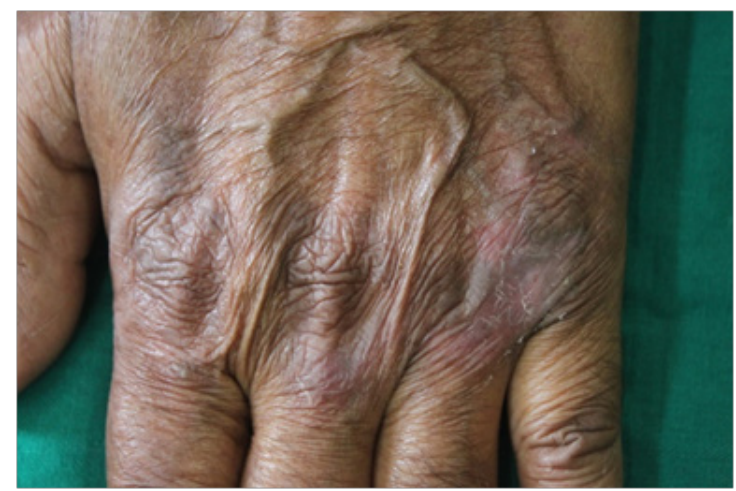

Figure I Erythema and erosions of the psoriatic plaques over dorsum on hand.

\author{
Volume 2 Issue 2 - 2018 \\ Deepak Vashisht, Prince Yuvraj Matharu, \\ Sukriti Baveja \\ Department of Dermatology, Pune University, India
}

Correspondence: Deepak Vashisht MD, Department of dermatology, Pune University, AFMC Pune, India, Tel +9190 4934 8800,Email deepak3975@gmail.com

Received: August 09, 2017 | Published: April 09, 2018

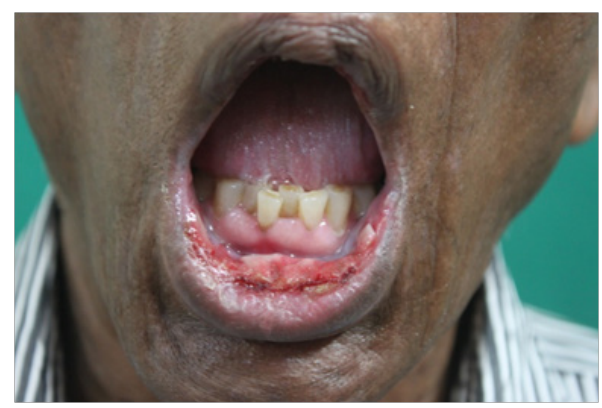

Figure 2 Superficial ulcerations and erosions on mucosal surface of both lips.

Laboratory studies revealed a hemoglobin of $11.9 \mathrm{~g} / \mathrm{dL}$, red blood cell count of $4.25 \mathrm{lacs} / \mu \mathrm{L}$, white blood cell count (WBC) of $7600 / \mu \mathrm{L}$ and the platelet count of $1,90,000 / \mu \mathrm{L}$. Serum biochemistry showed a serum creatinine level of $1.4 \mathrm{mg} / \mathrm{dL}$, blood urea nitrogen of $38.0 \mathrm{mg} /$ $\mathrm{dL}$, and total bilirubin of $0.9 \mathrm{mg} / \mathrm{dL}$ with direct bilirubin of $0.5 \mathrm{mg} / \mathrm{dL}$, SGOT of 37IU/L, SGPT of 20IU/L. Coagulation profile was within normal limits. Ultrasound studies of abdomen were normal.

Hence patient was diagnosed as methotrexate induced mucocutaneous ulceration. Methotrexate was stopped oral folic acid $5 \mathrm{mg}$ daily along with topical steroids and emollients were continued. The ulcerated skin lesions began to heal within 4days of discontinuing methotrexate and complete healing occurred after 14days. Patient was later started on biologics for Psoriatic arthritis by rheumatologist.

\section{Discussion}

Methotrexate (amethopterin) is an analog of folate which acts by inhibiting enzyme dihydrofolate reductase thereby inhibiting formation of tetrahydrofolate which is necessary in production of 1carbon units. This leads to inhibition of synthesis of thymidylate and purine nucleotides needed for RNA and DNA synthesis. Methotrexate continues to be one of the most effective drugs in management of Psoriasis and rheumatoid arthritis (RA) even after the advent of biologics for more than two decades owing to its efficacy, long track record of safety and cost factor. ${ }^{1,2}$ Side effect profile of Methotrexate vary from trivial to life threatening of which hepatotoxicity has been most feared. Extensive guidelines are in place for dosage, monitoring 
and follow up of treatment to avert any serious side effects. ${ }^{3}$ Cutaneous ulceration is one of the under reported and poorly characterized adverse event. Exact prevalence of this rare cutaneous manifestation is unknown due to under reporting.

Lawrence and Dahl categorized these cutaneous ulcerations in two types "Type I ulcers were superficial, painful and typically appeared in existing psoriatic plaques after a mean time lag of 10days from starting therapy at doses between 12.5 and 25 (median, 20)mg/ wk and healed rapidly (median, 10days). Type II ulcers occurred in clinically uninvolved skin, had a variable relationship to the duration of treatment at doses of 7.5 to 20 (median, 10)mg/wk and took a median of 9 weeks to heal". ${ }^{4}$ Exact etiology of ulceration in psoriatic plaques is unknown; probably it is either direct keratinocyte toxicity or increased mitotic activity in the plaques that makes them more susceptible. ${ }^{5,6}$ However this mechanism doesn't explain cutaneous ulcerations reported in a few patients without the history of psoriasis. ${ }^{7}$

Our patient presented with mucocutaneous ulcerations without fever, systemic symptoms, deranged biochemical parameters or features of pancytopenia. These ulcerations were more akin to Lawrence and Dahl type I ulcer as they developed in previous psoriatic plaques, appeared around 10 days of initiation of Methotrexate and healed promptly in a fortnight without scarring, albeit at a very low dose of $7.5 \mathrm{mg} / \mathrm{wk}$.

\section{Conclusion}

This case report reinforces the rare observation of mucocutaneous ulcerations even on low dose Methotrexate. Ulcerations can appear both at previously involved and uninvolved sites. These can be harbinger of sinister systemic adverse events. Prompt cessation of therapy can avert impending serious outcome. Physicians using Methotrexate should be watchful of cutaneous and oral ulcerations.

\section{Acknowledgments}

None.

\section{Conflicts of interest}

The authors declared that there are no conflicts of interest.

\section{References}

I. Bangert CA, Costner MI. Methotrexate in dermatology. Dermatol Ther. 2007;20(4):216-228.

2. Kurian A, Haber R. Methotrexate-induced cutaneous ulcers in a nonpsoriatic patient: case report and review of the literature. J Cutan Med Surg. 201 I; I5(5):275-279.

3. Chakravarty K, McDonald H, Pullar T, et al. BSR/ BHPR guideline for disease-modifying anti-rheumatic drug (DMARD) therapy in consultation with the British Association of Dermatologists. Rheumatology. 2008;47(6):924-925.

4. Lawrence CM, Dahl MG. Two patterns of skin ulceration induced by methotrexate in patients with psoriasis. J Am Acad Dermatol. 1984; I (6): I059-1065.

5. Reed KM, Sober AJ. Methotrexate-induced necrolysis.J Am Acad Dermatol. 1983;8(5):677-679.

6. Hoffman TE, Watson W. Methotrexate toxicity in the treatment of generalized pustular psoriasis. Cutis. 1978;2I(I):68-7I.

7. Ben-Amitai D, Hodak E, David M. Cutaneous ulceration: an unusual sign of methotrexate toxicity first report in a patient without psoriasis. Ann Pharmacother. 1998;32(6):65I-653. 